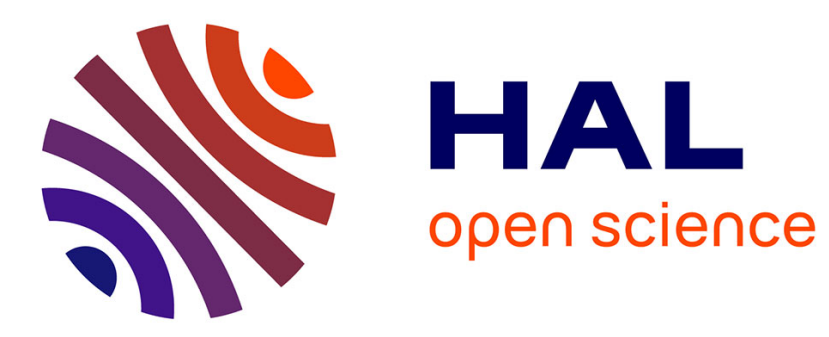

\title{
Prescription culturelle
}

Catherine Dutheil-Pessin, François Ribac

\section{To cite this version:}

Catherine Dutheil-Pessin, François Ribac. Prescription culturelle. Publictionnaire. Dictionnaire encyclopédique et critique des publics, 2018. hal-01927305

\section{HAL Id: hal-01927305 https://hal.science/hal-01927305}

Submitted on 19 Nov 2018

HAL is a multi-disciplinary open access archive for the deposit and dissemination of scientific research documents, whether they are published or not. The documents may come from teaching and research institutions in France or abroad, or from public or private research centers.
L'archive ouverte pluridisciplinaire HAL, est destinée au dépôt et à la diffusion de documents scientifiques de niveau recherche, publiés ou non, émanant des établissements d'enseignement et de recherche français ou étrangers, des laboratoires publics ou privés. 


\section{ublictionnaire}

Dietionnaire encyclopédique et eritique des Publies

\section{Prescription culturelle}

\section{Catherine Dutheil-Pessin et François Ribac}

Référence électronique

Catherine Dutheil-Pessin et François Ribac, Prescription culturelle. Publictionnaire. Dictionnaire encyclopédique et critique des publics. Mis en ligne le 09 avril 2018. Accès : http://publictionnaire.huma-num.fr/notice/prescription-culturelle/.

Le Publictionnaire. Dictionnaire encyclopédique et critique des publics est un dictionnaire collaboratif en ligne sous la responsabilité du Centre de recherche sur les médiations (Crem, Université de Lorraine) ayant pour ambition de clarifier la terminologie et le profit heuristique des concepts relatifs à la notion de public et aux méthodes d'analyse des publics pour en proposer une cartographie critique et encyclopédique.

Accès : http://publictionnaire.huma-num.fr/

Cette notice est mise à disposition selon les termes de la licence Creative Commons Attribution - Pas d'utilisation commerciale - Pas de modification 3.0 France.

Pour voir une copie de cette licence, visitez http://creativecommons.org/licenses/by-nc-

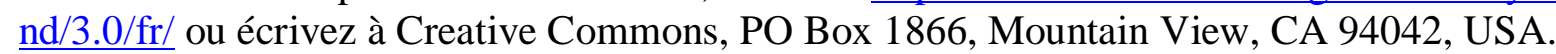

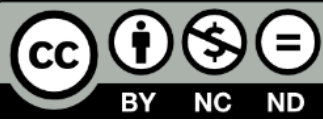




\section{Prescription culturelle}

Tout au long des $\mathrm{XIX}^{\mathrm{e}}$ et $\mathrm{XX}^{\mathrm{e}}$ siècles, un processus de constitution et de légitimation de corps d'experts professionnels s'est approfondi dans les sociétés industrialisées, notamment dans ce qu'on appelle communément sphère culturelle ou encore art, entendu ici comme le mouvement par lequel des biens, des activités et des espaces ont été désignés comme spécifiques et valorisés (Moulin, 1995 ; Shiner, 2001). Dans la France contemporaine, un ensemble de personnes et de dispositifs sont chargés de conseiller et de former (dans le sens d'éduquer et de donner forme) les publics, pour les orienter dans la nébuleuse culturelle et artistique : critiques, historien-ne-s de l'art, commissaires d'expositions, programmateurs de spectacles, acteurs des politiques culturelles, galeries, musées, bibliothèques, lieux de représentations, éditions (musicales), presse, libraires, fondations, médias, mais aussi l'État ou l'école (Leroy, 1990 ; Dubois, 1999 ; Waresquiel, 2001 ; Poirrier, 2002, 2010 ; Wallach, 2006 ; Goetschel, Yon, 2008 ; Urfalino, 1996 ; Charle, 2008 ; Guichard, 2011 ; Moulinier, 2011 ; Saez 2012). Une galaxie de recommandations, d'institutions et de dispositifs a donné lieu à de nombreuses déclinaisons selon les configurations locales et les périodes historiques, et à d'incessantes reconfigurations. Par des compétences reconnues, ces experts font autorité au sein d'institutions ou d'entreprises culturelles en produisant des jugements via des actions de prescription (Debruyne F., Pirolli F., à paraître) et, pour certains, en intervenant directement au sein des marchés de biens culturels (par exemple, en acquérant des toiles pour des musées ou en achetant et produisant des spectacles pour des festivals). Ce faisant, ils produisent et promeuvent des conceptions de l'art et de la culture. Simultanément, on sait que des amateurs - entendus ici au double sens de passionnés et de non professionnels - savent s'organiser et créer les conditions de leur plaisir (individuellement, collectivement), devenir des experts de leur art ou discipline, se prescrire et prescrire à d'autres des œuvres et des façons de faire (Hennion, Maisonneuve, Gomart, 2000).

On prendra pour guide le fil de cette relation dialectique entre experts professionnels de la prescription et «experts profanes » de la prescription amateur. Après une première ébauche de définition de la notion de prescription, on verra comment travaille un corps spécifique de prescripteurs professionnels qui pèse d'un poids important dans le domaine culturel, à savoir les programmateurs de spectacles. Puis un passage par la sociologie économique permettra de comprendre comment les biens culturels, spécifiques parce que relativement singuliers, trouvent place dans des marchés dédiés (production, circulation, consommation), lesquels sont régulés et d'une certaine manière organisés par des prescripteurs. On évoquera ensuite quelques-unes des questions essentielles liées à la numérisation et à l'abondance de biens culturels, à la mise en place d'une recommandation automatisée et à l'irruption d'une prescription amateur en ligne.

\section{La prescription}

Du latin praescribere: de prae, avant, et scribere, écrire (Littré). Historiquement, le terme vient du domaine juridique, la prescription désigne en effet l'écoulement d'un délai prévu par la loi, au-delà duquel une action judiciaire ne peut plus être exercée. Le terme renvoie aussi à la médecine, désignant au sein d'une relation professionnelle de soins l'acte, document écrit par le médecin, qui formalise et valide la délivrance de conseils et d'actions thérapeutiques (médicaments, soins, dispositifs) auprès d'un patient, l'ordonnance médicale étant née officiellement en 1322 par un édit royal. Si on laisse de côté l'importance significative de 
l'écriture (restriction en tête de la formule que le prêteur romain adressait au juge; ordonnance médicale sous forme d'un écrit normé et encadré sur le plan éthique), ce qui passe ici dans la prescription culturelle, c'est l'idée de conseil, de recommandation, de jugement, qui se fait généralement sous une forme sans contrainte, mais qui peut cependant prendre des voies se parant d'autorité.

Dans sa synthèse de la livraison de la revue Territoires contemporains intitulée «La prescription culturelle en question » (à paraître en 2018 et faisant suite au colloque du même nom organisé en avril 2017 à la Maison des sciences de l'homme [MSH] de Dijon par le Groupe d'étude de la prescription, accès : https://www.fabula.org/actualites/colloqueinternational-la-prescription-culturelle-en-question_78239.php), Pierre Delcambre a défini la prescription « comme une prétention sociale à faire faire (que l'on ait été sollicité ou non), qui suppose une connaissance préalable, une expertise spécifique et une connaissance ajustée de celui à qui l'on prescrit ». Cette activité s'exprime dans le cadre d'une relation le plus souvent top down et elle est soutenue par différents types d'argumentaires visant à convaincre (explications, recommandations, documents de communication à la rhétorique stabilisée). Elle implique aussi des dispositifs (bâtiments ou terrains pour des spectacles par exemple) et des outils communicationnels (programmes de saison pour une salle de spectacles...) par lesquels ces prescriptions sont délivrées et - le cas échéant - suivies par ceux et celles à qui elles sont destinées. En bref, prescrire suppose un certain type de relation avec ceux à qui l'on destine ses recommandations, et cette relation est organisée, normée, et équipée. La recherche de la qualité, de l'authenticité, de la nouveauté, engagent des jugements de goût, et d'une certaine façon la subjectivité des prescripteurs, mais une subjectivité élargie, informée, comme on va le voir avec l'exemple des programmateurs-trices de spectacles subventionnés.

\section{L'exemple de la programmation de spectacles subventionnés}

La politique culturelle, telle qu'elle est déclinée par les différentes incarnations de l'action publique en France, comprend plusieurs axes, tels que les musées, le patrimoine architectural, le soutien aux industries culturelles, etc. Toutefois, sa partie la plus visible est sûrement ce que l'on appelle «spectacle vivant», une politique qui privilégie la professionnalisation des équipes (artistiques, techniques, administratives) et des équipements consacrés aux spectacles. Partant, l'offre subventionnée de spectacles est de plus en plus souvent désignée par toutes sortes de personnes et d'institutions comme « la culture » à laquelle on assigne une mission émancipatrice, citoyenne, (re)créatrice de lien social (Rancière, 2008). Au cœur de cette rhétorique et de sa mise en pratique, les programmateurs-trices, experts ès spectacles, sont chargé-e-s de choisir et d'agencer de bonnes représentations (Dutheil-Pessin, Ribac, 2017). Leur rôle est crucial non seulement parce qu'il leur appartient de sélectionner, et donc d'éliminer, les propositions artistiques mais aussi parce que leur travail consiste à convertir des formes locales, un lieu, un artiste, une œuvre spécifique, en intérêt commun.

L'enquête que nous avons menée en 2011-2013 montre que les choix de programmation doivent prendre en compte une série de contraintes, organisationnelles, réglementaires, techniques, budgétaires, politiques, locales. Cela ne veut pas dire que le goût des programmateurs-trices n'est pas mobilisé, qu'il ne compte pas dans les choix, mais qu'il doit être balancé avec l'environnement et prendre en compte aussi bien l'élu à la culture, que la jauge, le bassin de population, la concurrence aux alentours, le budget dont on dispose, la presse, ou ce que l'on a déjà programmé. De fait, la programmation d'un spectacle, d'un trimestre, d'une saison ou d'un festival, sont le résultat d'une série de négociations aussi bien tacites qu'explicites.

Si l'on s'intéresse à la façon dont les spectacles sont repérés et sélectionnés, on doit mentionner la centralité des réseaux de professionnel-le-s, le terme réseau étant ici employé dans son acception commune. En effet, c'est par ces voies que l'on est informé sur ce qui 
émerge de nouveau, que l'on maintient ses connaissances techniques et institutionnelles à niveau, c'est dans ce cadre que l'on échange avec des collègues et que l'on trouve des appuis pour choisir - voire coproduire - «ses » spectacles. Si les différents outils de communication (courrier, téléphone, Web) et les spectacles auxquels on assiste sont des sources d'information, les réunions organisées par les réseaux professionnels et la discussion informelle avec les collègues, par exemple lors des festivals, sont tout aussi essentiels. Les divers et très nombreux réseaux de professionnels disciplinaires, territoriaux, thématiques, constituent des espaces de qualification des œuvres, où se construit collectivement et de façon interactionnelle la réputation des spectacles, des artistes... et des prescripteurs eux-mêmes. Un point qui confirme ce que la sociologie des sciences a montré à propos de l'expertise (Trépos, 1996 ; Collins, Evans, 2007) : le savoir des individus, y compris celui des experts, est fondamentalement lié aux informations (textes, réunions, supports, rumeurs, etc.) qui circulent au sein de leur groupe social. Et s'il bénéficie de relais institutionnels suffisamment forts et est capable de faire valoir ses méthodes comme les seules possibles, ce groupe social acquiert un pouvoir d'énonciation et une autorité. De nombreuses collectivités locales présentent depuis quelques années des spectacles qu'elles subventionnent dans le cadre du festival d'Avignon, précisément parce qu'elles ont bien compris l'importance de s'attacher ces réseaux de programmateurs et les événements qui les fédèrent. Elles se positionnent ainsi comme des acteurs essentiels dans le processus de qualification des spectacles et comme des opérateurs incontournables de la politique culturelle.

En régime républicain, les programmateurs-trices de spectacles subventionnés et leurs divers réseaux bénéficient d'une délégation que l'on pourrait comparer à celle que l'on accorde aux experts scientifiques dans l'agence du médicament ou aux élus: d'une part, ils-elles expertisent la «culture » et, d'autre part, on leur confie le mandat de décliner leurs choix dans l'espace public. Cependant, et comme on l'a noté plus haut, ce processus de sélection résulte d'une série de négociations au sein desquelles des (groupes de) personnes (spectateurs, élus, presse, collègues, équipes artistiques, etc.), des flux d'informations et des réseaux de communication interagissent. Même la prescription «par le haut» doit faire avec l'expertise des spectateurs ordinaires (Leveratto, 2000).

\section{Les marchés de la culture, marchés à prescripteurs}

Pourquoi les programmateurs et programmatrices, et plus généralement les prescripteurs culturels, occupent-ils une place si centrale ? Si la sphère culturelle a recours à des prescripteurs, c'est, selon Lucien Karpik (2007), en raison de la nature même des biens culturels. Ceux-ci sont en effet «singuliers », formant chacun une configuration particulière de qualités, qui tiennent à la fois aux créateurs, aux interprètes, à leur performance, à leurs techniques, à leur puissance d'évocation, de signification ou d'émotion... Autant de paramètres, difficilement quantifiables et appréciables au préalable, qui font du monde de l'art un monde d'entités uniques, inéquivalentes. Ces biens sont recherchés pour cette singularité même. Un bien culturel présente donc à la fois un certain degré d'opacité et une incertitude liée au risque d'inadéquation avec les clients potentiels, opacité et incertitude que le prescripteur permet de réduire au mieux. Par une prescription de jugement (Hatchuel, 1995) qu'il va lui-même contribuer à énoncer et à former collectivement, le prescripteur fournit simultanément son jugement et la «bonne façon» de juger. Pour reprendre les mots de Lucien Karpik (2007), les marchés des biens culturels fonctionnent avec de multiples «dispositifs de jugement», qui se mêlent, se complètent ou se concurrencent. La relation acheteurs/prescripteurs est une relation reposant sur la confiance dans les connaissances de l'expert, dans son savoir-faire, dans ses compétences de connaisseur, mais aussi dans son indépendance ou sa neutralité. Cette confiance peut dans certaines circonstances virer en défiance, et en contestation du savoir du prescripteur (on y reviendra plus loin). De plus, « en 
permettant à l'acheteur de s'orienter vers un offreur ou en le dissuadant d'aller vers certains, en structurant sa représentation des biens à rechercher, le prescripteur contribue à la formation de la valeur et modifie le champ des échanges, donc le marché lui-même » (Hatchuel, 1995 : 218). En d'autres termes, les dispositifs de prescription culturelle contribuent, de façon variable mais patente, à la structuration même du marché des biens culturels, une situation déjà mentionnée à propos de la programmation de spectacles.

\section{Les systèmes de recommandation algorithmique à l'heure de l'hyper-choix et du Web}

Avec l'essor du Web, de plus en plus de consommateurs s'informent sur la toile avant d'acquérir un bien culturel, de se rendre à un spectacle ou à une exposition. Dans le même temps, de nombreux contenus - de la musique, des séries télévisées, des livres papiers ou électroniques (Pirolli, 2015) - sont fréquemment, voire exclusivement, disponibles en ligne (Debruyne, 2012). Enfin, le nombre de contenus disponibles en ligne est littéralement incommensurable, et l'hyper-choix est devenu la règle (Chapelain et al., à paraitre). Dans cette configuration (Cnil, 2015), la recommandation et la personnalisation sont un impératif marketing de la relation au client. Il s'agit de se positionner avantageusement par rapport aux concurrents, en valorisant le service rendu : devenir prescripteur peut alors permettre aux fournisseurs d'accès et de contenus, qui peuvent être les mêmes, de s'imposer sur le marché. On distingue trois types de recommandation en ligne :

- Une prescription amateur délivrée par les internautes, en particulier par l'intermédiaire de blogs et des réseaux sociaux, horizontale (Bouquillion, Matthews, 2010 ; Pasquier, 2014 ; Béliard, 2014 ; Ribac, 2010, à paraître).

- Une prescription éditoriale et/ou professionnelle, plus verticale, délivrée par les instances et dispositifs mentionnés au début de cette notice (programmateurs, commissaires d'exposition, musées, salles de spectacles, presse, média, etc.).

- Une prescription algorithmique, personnalisée grâce à un moteur de recommandation (Kembellec, Chartron, Saleh, 2014 ; Cardon, 2015).

Ces trois types peuvent bien entendu s'hybrider.

La constitution d'immenses banques de données (big data, création d'une industrie de la donnée) a permis l'existence de développeurs de services de recommandation qui touchent tous les secteurs de la culture, et qui vont aussi bien au-delà. Ces systèmes de recommandation sont fondés sur l'alliance de la quantification statistique et de la qualification documentaire. Les données ainsi collectées ont souvent une traçabilité à sens unique, c'est-àdire qu'elles ne sont pas consultables par les usagers. Les données collectées en ligne se répartissent en données explicites fournies, consenties par l'internaute, et en données implicites, extraites des traces de l'activité de l'internaute. Il existe six groupes de données :

- personnelles ;

- descriptives, attachées au contenu (métadonnées) ;

- de popularité (par les like...) ;

- d'enrichissement (fournies par des internautes et des professionnels) ;

- comportementales et de goût, permettant de profiler l'internaute usager ;

- celles issues d'objets connectés, permettant d'avoir des indications sur l'état émotionnel de l'usager (notamment dans le secteur du jeu vidéo...).

Si l'on prend l'exemple de la musique, les données sur les internautes sont de plus en plus constituées grâce à une contextualisation automatisée du contenu de l'activité d'un individu ou d'un collectif, d'un partage, d'une compilation de titres (Fanen, 2017). La plateforme de streaming Spotify a ainsi racheté The Echo Nest, une société qui mesure «l'acousticité » et la «danceabilité » des chansons écoutées par les internautes afin de proposer des playlists en fonction des habitudes et des humeurs des utilisateurs. On s'oriente donc vers une prédiction plus personnalisée, des services ambiants cherchant à favoriser un engagement plus fort entre 
les individus et les œuvres (par des fictions plus immersives et plus interactives, ainsi que le développement de contenus narratifs sur plusieurs médias). Cette évolution n'est pas sans susciter des inquiétudes... Ghislaine Chartron (à paraître) évoque ainsi un "pacte faustien » entre les industries culturelles et la recommandation algorithmique. Pour ce qui concerne le domaine de la critique et du jugement en ligne (pour les sites consacrés aux films par exemple), les algorithmes servent surtout, selon Dominique Pasquier (2014), à construire à moindres frais des modèles commerciaux rentables, à travers une idéologie de la participation et de la créativité. De même, le fait que l'activité bénévole des internautes soit mobilisée, le plus souvent à leur insu, afin de modéliser des comportements et nourrir les algorithmes de recommandation pose de nombreuses questions éthiques et politiques qui ont notamment été relevées par les critiques du digital labor (Scholz, 2013 ; Fuchs, 2014 ; Dujarier, 2008 ; Cardon, Casilli, 2015). Cependant, étudiant l'économie de la découverte chez les amateurs de musique à l'ère numérique, les recherches de Sophie Maisonneuve (2017) montrent que ceuxci piochent dans un « répertoire de dispositifs de recommandation» qui se combinent et s'enchâssent. Parmi ceux-ci, les échanges entre pairs experts, reposant sur la connaissance mutuelle et la confiance, permettent l'échange de conseils éclairés et peuvent s'articuler avec des suggestions algorithmiques. Dans un même ordre d'idées, on pourrait noter que la boîte noire des programmateurs (humains) n'est pas moins obscure que celle des algorithmes (nonhumains, mais programmés par des humains)...

\section{La prescription amateur : une menace ?}

Avec l'apparition des réseaux de Peer to Peer puis des plate-formes collaboratives du Web 2.0 (blogs, forums de discussion, sites de partage de vidéos et/ou de photos, réseaux sociaux), la prescription culturelle amateur a acquis une visibilité certaine (Allard, 2007 ; Beuscart et al., 2009 ; Flichy, 2010 ; Ribac, 2010, à paraître). Des passionné-e-s de tous les continents ont ainsi commencé à critiquer, discuter et mettre à disposition des autres, gratuitement, des contenus culturels : musiques, films, séries TV, jeux vidéos, ebooks, photos, etc. D'autres, ou les mêmes, ont chroniqué au jour le jour des concerts, expositions, rétrospectives, pièces de théâtre, opéras, etc. Certaines de ces plate-formes et/ou de ces contributeurs ont été capables d'acquérir une grande notoriété. Au moment où ces lignes sont écites, les Youtubeurs-euses complètent désormais le paysage de la prescription culturelle en ligne, et certain-e-s sont suivi-e-s par des milliers d'internautes (Beuscart, Mellet 2015). Jadis réservées aux critiques et aux journaux professionnels, ces activités n'ont pas seulement été rendue possible parce que les nouveaux critiques peuvent toucher de vastes audiences mais aussi parce que les ressources disponibles sur la Toile permettent, à condition de bien maîtriser les outils numériques et les moteurs de recherche, de dénicher des trésor oubliés, puis de les défendre et les promouvoir.

Car l'un des aspects le plus marquants de cette prescription amateur est certainement la part patrimoniale, parfois même quasi archéologique, des contenus qui sont présentés, débattus et distribués par la prescription amateur en ligne. La Longue Traîne (Anderson, 2006) avait proposé l'idée que, débarrassés de la contrainte de présenter des produits dans des magasins, des sites de vente en ligne pourraient stocker un grand nombre de références dans des entrepôts et gagner de l'argent en vendant de toutes petites quantités d'un très grand nombre d'articles. Mais ce que l'on n'avait pas véritablement vu venir, c'est que la numérisation des contenus - en particulier la musique et les films - allait permettre aux internautes de partager, d'échanger et de vanter les mérites de répertoires qui n'étaient même plus commercialisés et de construire des espaces d'échanges non marchands. Pour apprécier les effets de cette prescription amateur en ligne sur la prescription professionnelle (en particulier la critique musicale et cinématographique issue de la presse écrite et audiovisuelle), il faut prendre en compte cette relativisation de «l'actualité culturelle». Toutefois, après plus de dix ans de 
Web 2.0, il est patent que le monde professionnel n'a pas été terrassé par l'expertise et la prescription amateur. Le Peer to Peer - dont on oublie trop souvent qu'il est aussi une sorte de prescription - a bien forcé l'industrie discographique et musicale à trouver d'autres débouchés (la scène et le streaming) et à redéfinir ses périmètres d'activités (Guibert, SagotDuvauroux 2013). La critique amateur en ligne a certainement limité le pouvoir des critiques professionnels (un film est-il encore ruiné par une mauvaise critique dans le quotidien Libération ?), les réseaux sociaux permettent régulièrement à des artistes d'apparaître voire de réapparaître, des chapitres entiers de l'histoire des arts et donc de la qualité esthétique sont ébauchés et réinventés en ligne. Cependant, les académiques fabriquent encore des histoires de la musique et de l'esthétique, tandis que la presse chronique toujours l'actualité et que les programmateurs de spectacles et de festivals voient leurs salles se remplir. Autrement dit, la révolution "numérique" (terme fourre-tout et confus) que prophétisaient les modernes n'a pas eu lieu dans les termes où on nous l'annonçait. Quelle que soit leur vigueur, les Pro Ams, les amateurs qui adoptent des normes et des rythmes quasi professionnels et notamment en matière de prescription culturelle, n'ont pas ruiné les fondations et les institutions prescriptrices d'avant le Web ; ils ont déstabilisé l'édifice professionnel certes, mais celui-ci est encore là. Pour comprendre ce processus on peut avancer au moins trois raisons.

Premièrement et comme on l'a dit plus haut, l'hyper-choix, c'est-à-dire l'abondance de biens et d'informations disponibles sur la toile, nécessite de disposer d'encore plus de dispositifs de jugement pour s'orienter. Sous cet angle, la prescription amateur est venue renforcer ceux qui existaient avant le Web. Hyper-choix culturel = prescriptions hyper-diversifiées !

Deuxièmement, de la même façon qu'une frange des consommateurs se méfie des enseignes et des modèles dominants, par exemple lorsque les grandes surfaces se convertissent au bio (Hirschman, 1970), une partie des usagers considère avec méfiance le fait de confier à Google, à son smartphone ou à des plate-formes en ligne d'apprécier la qualité d'un bien culturel. Ils conservent leur confiance au «vieux monde ».

Troisièmement, les formes d'expertise et de recommandation amateur existaient déjà avant le Web. La visibilité des amateurs, leur prétendu sacre (Flichy, 2010), s'appuie au moins autant sur des pratiques domestiques et médiatiques antérieures que sur les potentiels du Web 2.0 et les ressources de l'intelligence collective connectée (Lévy, 1994). Depuis longtemps, les auditeurs faisaient connaître leurs opinions - en direct ou par le biais de courriers - à des émissions de radio, de télévision et des journaux. Depuis longtemps, des grands amateurs recommandaient à leurs proches des répertoires (Hennion, Maisonneuve, Gomart, 2000) et leur distribuaient des cassettes et des playlists. Depuis longtemps, des fans de jazz, de rock punk et de bien d'autres styles musicaux éditaient bénévolement des fanzines (Savage, 1991 ; Atton, Hamilton, 2008) qui vantaient des artistes ignorés par les grands médias et les historiens de l'art. Les usages du Web ont permis d'intensifier et de diversifier des pratiques qui préexistaient et qui, comme aujourd'hui, s'articulaient avec l'offre et les médias professionnels. En attribuant au «numérique» des capacités intrinsèques, on oublie l'antériorité de ces pratiques sociales. Une technologie (et les usages qui s'y développent) peut amplifier et soutenir de nouveaux usages mais elle ne crée rien d'entièrement nouveau ; au contraire, il faut que sa part de nouveauté soit réduite pour qu'elle ait des chances d'être efficiente (Edgerton, 2006).

Enfin, on peut remarquer que l'essor de la recommandation amateur en matière de culture peut être corrélé avec l'essor des formes profanes d'expertise qui ont émergé - tantôt sous la forme des lanceurs d'alerte (Chateauraynaud, Torny, 1999), tantôt sous la forme d'associations - sur des terrains aussi variés que l'énergie nucléaire, le sida (Dodier, 2003), les maladies rares, l'alimentation, les OGM, etc., depuis les années 1960 (Callon, Barthe, Lascoumes, 2001; Méadel, 2010). Dans un contexte où les conflits d'intérêts sont de moins en moins tolérés par la société et où la parole des experts est sensiblement décrédibilisée, on 
peut donc probablement interpréter l'essor de la prescription culturelle amateur comme l'une des expressions de cette aspiration à des formes plurielles et plus transparentes d'expertise.

\section{Bibliographie}

Allard L., 2007, «Émergence des cultures expressives, d'Internet au mobile », Mediamorphoses, 21, pp. 19-25.

Anderson C., 2006, La Longue Traîne. La nouvelle économie est là !, trad. de l'anglais par B. Vadé et M. Le Séac'h, Paris, Pearson, 2009.

Atton C., Hamilton J. F., 2008, Alternative Journalism. Journalism studies, key texts, Londres, Sage.

Béliard A.-S., 2014, « Jeux croisés entre critique amateur et critique professionnelle dans les blogs de séries télévisées », Réseaux, 183, pp. 95-121. Accès : https://www.cairn.info/revuereseaux-2014-1-page-95.htm.

Beuscart J.-S. et al., 2009, «Pourquoi partager mes photos de vacances avec des inconnus? Les usages de Flickr», Réseaux, 54, pp. 93-129. Accès : https://www.cairn.info/revuereseaux-2009-2-page-91.htm.

Beuscart J.-S., Mellet K., 2015, «La conversion de la notoriété en ligne. Une étude des trajectoires de vidéastes pro-am », Terrains \& travaux, 26, pp. 83-104. Accès : https://www.cairn.info/revue-terrains-et-travaux-2015-1-page-83.htm.

Bouquillion P., Matthews J. T., 2010, Le Web collaboratif. Mutations des industries de la culture et de la communication, Grenoble, Presses universitaires de Grenoble.

Callon M., Barthe Y., Lascoumes P., 2001, Agir dans un monde incertain. Essai sur la démocratie technique, Paris, Éd. Le Seuil.

Cardon D., 2015, À quoi rêvent les algorithmes. Nos vies à l'heure des big data, Paris, Éd. Le Seuil.

Cardon D., Casilli A., 2015, Qu'est-ce que le digital labor ?, Bry-sur-Marne, Ina Éd.

Chapelain B. et al., à paraître, La Prescription culturelle. Avatars et médiamorphoses, Villeurbanne, Presses de l'Enssib.

Charle C., 2008, Théâtres en capitales. Naissance de la société du spectacle à Paris, Berlin, Londres et Vienne, Paris, A. Michel.

Chartron G., à paraître, «Industries culturelles et recommandation algorithmique : un pacte faustien?», Territoires Contemporains.

Chateauraynaud F., Torny D., 1999, Les Sombres Précurseurs. Une sociologie pragmatique de l'alerte et du risque, Paris, Éd. de l'École des hautes études en sciences sociales.

Cnil, 2015, «Les données, muses et frontières de la création. Lire, écouter, regarder et jouer à l'heure de la personnalisation », Cahiers IP, 3 .

Collins H., Evans R., 2007, Rethinking Expertise, Chicago, University of Chicago Press.

Debruyne F., 2012, «Le disquaire et ses usagers. Du magasin au site Web », Communication \& langages, 173, pp. 49-65. Accès : https://www.cairn.info/revue-communication-etlangages1-2012-3-page-49.htm.

Debruyne F., Pirolli F., dirs, à paraître, «Prescription et recommandation : agir et faire 
agir ? » Études de communication, 49.

Delcambre P., à paraître, «La prescription culturelle quoi de particulier ? L'exemple des arts de la scène », Territoires Contemporains.

Dodier N., 2003, Leçons politiques de l'épidémie de sida, Paris, Éd. de l'École des hautes études en sciences sociales.

Dubois V., 1999, La Politique culturelle. Genèse d'une catégorie d'intervention publique, Paris, Belin.

Dujarier M.-A., 2008, Le Travail du consommateur. De MacDo à eBay: comment nous coproduisons ce que nous achetons, Paris, Éd. La Découverte, 2014.

Dutheil-Pessin C., Ribac F., 2017, La Fabrique de la programmation culturelle, Paris, Éd. La Dispute.

Edgerton D., 2006, The Shock Of The Old. Technology and Global History since 1900, Londres, Profile Books.

Fanen S., 2017, Boulevard du Stream. Du MP3 à Deezer, la musique libérée, Bègles, Éd. Le Castor astral.

Flichy P., 2010, Le Sacre de l'amateur. Sociologie des passions ordinaires à l'ère numérique, Paris, Éd. Le Seuil.

Fuchs C., 2014, Digital Labor and Marx, New York, Routledge.

Goetschel P., Yon J.-C., dirs, 2008, Directeurs de théâtre. XIX ${ }^{e}-X X^{e}$ siècles, Paris, Publications de la Sorbonne.

Guibert G., Sagot-Duvauroux D., 2013, Musiques actuelles, ça part en live. Mutations économiques d'une filière culturelle, Paris, Irma/DEPS.

Guichard C., 2011, «Les formes de l'expertise artistique en Europe (XIV ${ }^{\mathrm{e}}-\mathrm{XVIII}{ }^{\mathrm{e}}$ siècle) », Revue de synthèse, 132, 1, pp. 1-11.

Hatchuel A., 1995, «Les marchés à prescripteurs. Crises de l'échange et genèse sociale », pp. 205-225, in: Jacob A., Vérin H., dirs, L’Inscription sociale du marché, Paris, Éd. L'Harmattan.

Hennion A., Maisonneuve S., Gomart É., 2000, Figures de l'amateur. Formes, objets, pratiques de l'amour de la musique aujourd'hui, Paris, Éd. La Documentation française.

Hirschman A. O., 1970, Défection et prise de parole. Théorie et applications, trad. de l'anglais par C. Besseyrias, Paris, Fayard, 1995.

Karpik L., 2007, L'Économie des singularités, Paris, Gallimard.

Kembellec G., Chartron G., Saleh I., dirs, 2014, Les Moteurs et systèmes de recommandation, Londres, Iste éd.

Leroy D., 1990, Histoire des arts du spectacle. Aspects économiques, politiques et esthétiques de la Renaissance à la Première Guerre mondiale, Paris, Éd. L'Harmattan.

Leveratto J.-M., 2000, La Mesure de l'art. Sociologie de la qualité artistique, Paris, Éd. La Dispute.

Lévy P., 1994, L'Intelligence collective. Pour une anthropologie du cyberspace, Paris, Éd. La Découverte.

Maisonneuve S., 2017, «L'économie de la découverte musicale à l'ère numérique : la 
performance des dispositifs de prescription par l'amateur», colloque international $L a$ prescription culturelle en question, Université de Dijon, 6 avr.

Méadel C., 2010, «Les savoirs profanes et l'intelligence du Web », Hermès. La Revue, 2, 57, pp. 111-117. Accès : https://www.cairn.info/revue-hermes-la-revue-2010-2-page-111.htm.

Moulin R., 1995, De la valeur de l'art. Recueil d'articles, Paris, Flammarion.

Moulinier P., 1999, Les Politiques publiques de la culture en France, Paris, Presses universitaires de France, 2010.

Pasquier D., 2014 «Les jugements profanes en ligne sous le regard des sciences sociales », Réseaux, 183, pp. 9-25. Accès : https://www.cairn.info/revue-reseaux-2014-1-page-9.htm.

Pirolli F., dir., 2015, Le Livre numérique au présent. Pratiques de lecture, de prescription et de médiation, Dijon, Éditions universitaires de Dijon.

Poirrier P., dir., 2002, Les Politiques culturelles en France, Paris, Éd. La Documentation française.

Poirrier P., dir., 2010, Politiques et pratiques de la culture, Paris, Éd. La Documentation française.

Rancière J., 2008, Le Spectateur émancipé, Paris, Éd. La Fabrique.

Ribac F., 2010, « Ce que les usagers et Internet font à la prescription culturelle publique et à ses lieux : l'exemple de la musique en Île-de-France », Programme interministeriel Culture et Territoires en Île- de-France [rapport]. Accès : https://hal.archives-ouvertes.fr/hal01327071/document.

Ribac F., à paraître, «Amateurs et professionnels, gratuité et profits aux premiers temps du Web 2.0 : l'exemple de la blogosphère musicale », Transposition.

Saez G., dir., 2012, Les Nouveaux Enjeux des politiques culturelles. Dynamiques européennes, Paris, Éd. La Découverte.

Savage J., 1991, England's Dreaming. Sex Pistols and Punk Rock, Londres, Faber.

Scholz T., ed., 2013, Digital Labor. The Internet as Playground and Factory, New York, Routledge.

Shiner L., 2001, The Invention of Art. A Cultural History, Chicago, University of Chicago Press.

Trépos J.-Y., 1996, La Sociologie de l'expertise, Paris, Presses universitaires de France.

Urfalino P., 1996, L'Invention de la politique culturelle, Paris, Hachette, 2004.

Wallach J.-C., 2006, La Culture, pour qui? Essai sur les limites de la démocratisation culturelle, Toulouse, Éd. de l'Attribut.

Waresquiel E. de, 2001, Dictionnaire des politiques culturelles de la France depuis 1959, Paris, Larousse/CNRS Éd. 